\title{
s. \\ Enhanced Techniques for PDF Image Segmentation and Text Extraction
}

\author{
${ }^{1}$ Madhuri Patil, ${ }^{2}$ Monika Pune, ${ }^{3}$ Ajay Zaware, ${ }^{4}$ A.D. Kulkarni \\ 1,2,3,4Electronics and Telecommunication Department, Savitribai Phule Pune University \\ Email: ${ }^{1}$ patil.madhuri42@gmail.com, ${ }^{2}$ md.pune2013@gmail.com, ${ }^{3}$ a.m.zaware@gmail.com, \\ 4aniketkulkarni87@yahoo.com
}

\begin{abstract}
Extricating content articles from the PDF pictures is a trying issue. The substance data show in the PDF pictures contains certain significant information for programmed explanation, ordering and so on. However, assortments of the substance due to contrasts in substance style, textual style, estimate, introduction, arrangement and additionally complex make the issue of modified substance extraction to an extraordinary degree troublesome and testing work. This paper presents two strategies under square based portrayal. After a concise presentation of the arrangement strategies, two techniques were improved and results were assessed. The execution estimations for division and time usage are striven for both the models.
\end{abstract}

Keywords - PDF, Extraction, Segmentation, Enhancement

\section{INTRODUCTION}

With the unprecedented movement in Computer Technology and correspondence innovation, the forefront society is entering to the information edge. In change in the routine record structure, people now take after electronic chronicle system (PDF Format) for correspondence and limit which is by and by fundamental. In any case, on complex matters, the report picture is difficult to decisively perceive the information particularly out of the need.

On such cases preprocessing the record is done before its passage. Picture division theory, as cutting edge picture get ready has transformed into a basic bit of individual's dynamic research. Picture dealing with report picture division speculation is a basic research subject in the process it is overwhelmingly between the record picture pre-planning and moved character affirmation a basic association between.

The respectably feasible and normally used for report picture division and portrayal methodologies join edge, and geometric examination and distinctive groupings. Resulting to dividing, Text part is perceived and evacuated for further process, sometime recently, content extraction techniques has been made just on monochrome documents [1]. These methodologies can be named base up, top-down and cross breed.
Later with the growing necessity for shading files, strategies [2] have been proposed the division frameworks like Block based picture division [3] is used broadly before long. Under this square based division, the examination goes (i) $\mathrm{AC}$ Coefficient Based procedure and (ii) Histogram based methodology this paper is dealt with as takes after: In region II the short introduction of PDF Image. (iii) It talks about the review of piece based division.

\section{PROBLEM STATEMENT}

To Extract text objects from PDF image uses two block based classification and method contains in it is $\mathrm{AC}$ coefficient based technique and $\mathrm{K}$ means clustering. These techniques also evaluated performance metrics for segmentation and time consumption are tested for both the models.

\section{MOTIVATION}

In Existing approach for convincing substance extraction from graphical report pictures. The calculation Morphological Component Analysis (MCA) estimation, a movement of scanty portrayal framework with two appropriately picked discriminative over total lexicons. Two discriminative word references relied on upon undecimated wavelet change and curvelet change.

\section{EXISTING SYSTEM}

In Existing methodology for compelling content extraction from graphical report pictures. The calculation utilized Morphological Component Analysis (MCA) calculation, a progression of inadequate representation structure with two suitably picked discriminative over total lexicons. Two discriminative word references depended on Undecorated wavelet change and curvelet change. This technique touching amongst content and representation and furthermore coldhearted to various text style styles, sizes, and introductions. S.Audithan et.al detailed an effective and computationally quick technique to concentrate content districts from records. They proposed Haar discrete wavelet change to recognize edges of hopeful content locales. Non-content edges were 
evacuated utilizing thresholding method. They utilized morphological expansion administrator to interface the disengaged competitor content edge and after that a line include vector chart was produced in view of the edge outline. This strategy misused an enhanced watchful edge identifier to identify content pixels. The stroke data was separated the spatial conveyance of edge pixels. At long last content districts were created and sifted by line highlights.

\section{PROPOSED SYSTEM}

\section{A. Image Acquisition}

The Image Acquisition is the process of collection of images for picture and text segmentation in PDF image document. Image acquisition is used to scanned image for picture and text segmentation in image document.

\section{B. Image preprocessing}

Image preprocessing is used to improve image data so that it removes undesired distortions and/or it enhances image features that are relevant for further processing. Image processing helps to identify colors, shades and relationships which cannot be perceived by the human eye. The identification problems like blurred text data, noisy data in document images are solved by image processing. Image processing is used for analyze and manipulation of digitized images which improves the quality of images. The color images are then converted to grey level images by using the following formula grey $(i, j)=0.59 \operatorname{green}(i, j)+0.30$ $\operatorname{red}(i, j)+0.11$ blue $(i, j)$.

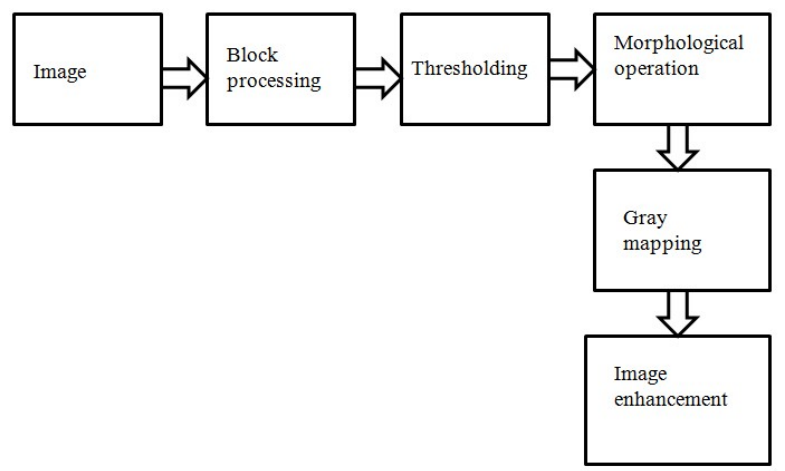

Figure 1: Block diagram of the proposed system

\section{Block processing}

Images can either be too large to load into memory, or else they can be loaded into memory but then be too large to process hence block processing is used to divide the input image into number of blocks as per the user- specified size, and It processes each block individually and then reassembles each block results into the output image. If we want to divide an input image into blocks and process each block Individually, the function blkproc is used that allow to process distinct blocks.

\section{G. Post Processing}

Post processing endeavors on increment the nature of a veil picture. Post handling is performed with the assistance of Morphology. The morphological operations are widening and disintegration. Dilation is nothing but addition of pixels to the boundaries of objects in an image, while subratction pixels from the boundaries of objects in an image comes under erosion. The quantity of pixels included or expelled from the items in a picture relies on upon the size and state of the organizing component used to prepare the image.

\section{SYSTEM ARCHITECTURE PDF IMAGE MECHANISM}

PDF format is converted into images using available commercial software's so that each PDF page is converted into image format. From that image format the text part are segmented and extracted for further process.

\section{BLOCK BASED SEGMENTATION}

To change and rearrange the representation of a picture into something which is more important and less demanding to break down is called as block based segmentation. Image division is commonly used to divide one image into another to find articles and limits (lines, bends, and so forth.) in images. All the more accurately, picture division is the way toward doling out a name to each pixel in a picture to such an extent that pixels with a similar mark share certain visual qualities.

A large portion of the current explores in this field for most of the parts in view of either layer based or piece based. This piece based division approach isolates a picture into squares of districts (Fig:1). Every district takes after surmised protest limits, and is made of rectangular squares. The extent of the squares may change inside a similar area to better rough the genuine protest limit.

Piece based division calculations are produced for most of the parts in grayscale or shading compound picture, For instance, In [4], content and line design are removed from check pictures. In [5], proposed square based clustering algorithm, [6] propose a characterization calculation, in light of the edge of the quantity of hues in every piece. [7], approach in view of accessible nearby surface components [8], detection using mask [9], and block classification algorithm for effective coding by thresholding DCT energy [10].

\section{TEXT EXTRACTION}

By applying the two strategies of block based technique, the picture is segmented into

\section{Smooth region (Background)}

2. Non Smooth district

3. Text districts

4. Image region 
It demonstrates that while dividing the PDF picture, foundation is distinguished as smooth squares. The frontal area ( non- smooth piece), utilizing K-implies calculation the content and picture squares are portioned and in this way message part is isolated or removed from the PDF picture. In the system, the PDF picture is portioned into $16 \times 16$ pieces, and afterward a histogram circulation for every pixel in each fragmented gathering is registered. Gathering of pixels is finished.

\section{RESULT ANALYSIS}

Input files:
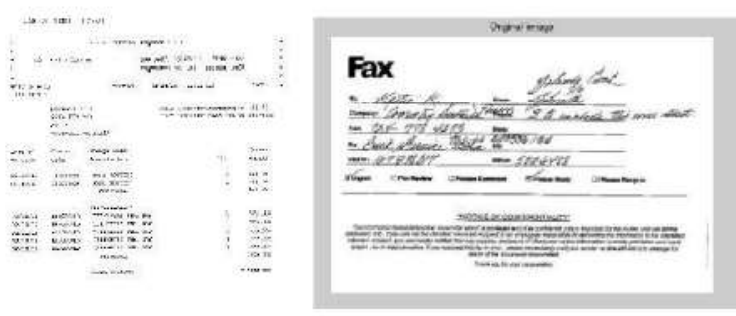

Figure 2: PDF to Image Conversion

Gray Conversion:

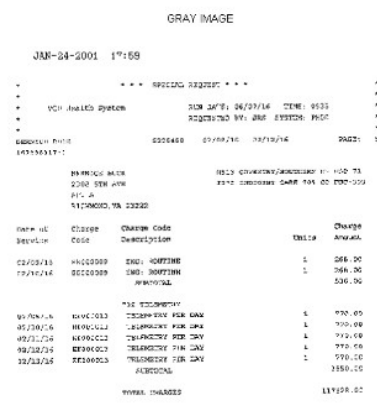

Figure 3: Gray Image

Segmentation:

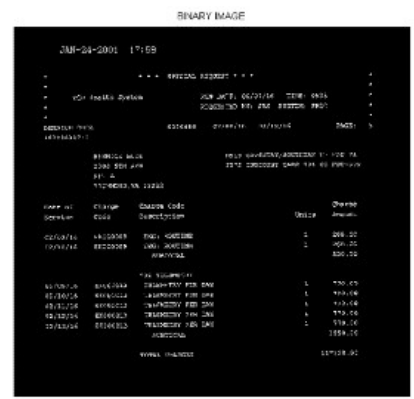

Figure 4: Binary Image

Morphological Operation:

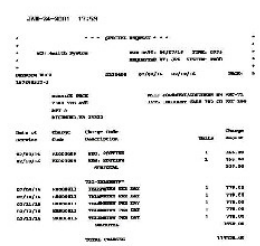

Figure 5: Eroded Image

Image Enhancement Operations:

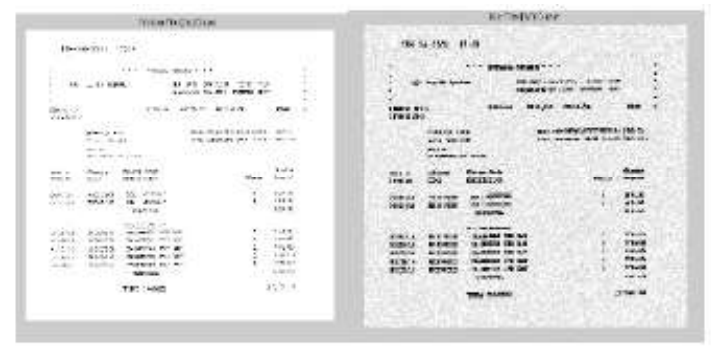

Figure 6: Noise Removal in an Image

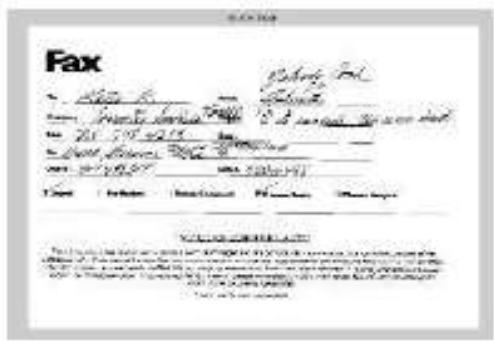

Figure 7 : Contrast and Brightness Adjusted Image

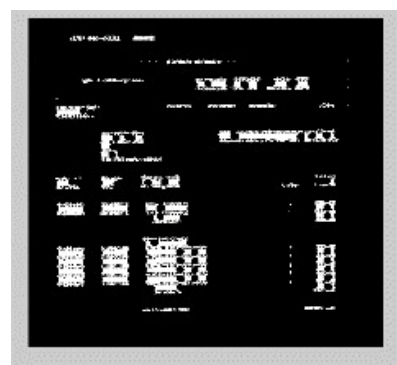

Figure 8: Translated Image

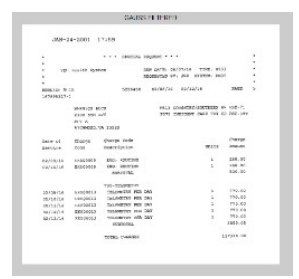

Figure 9: Filtered Image

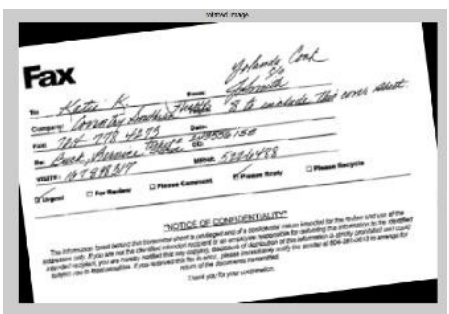

Figure 10: Tilting of image

\section{CONCLUSION}

This paper helps people to achieve higher character recognition rate and also used to improve the quality of image. This paper include the advantage and disadvantage of both the algorithms, from the performance analysis, If the user want to trade a little 
time for a better accuracy, then the AC Coefficient based technique will be suitable. However if the user requires quick retrieval and want to tolerate a slightly less reliable outcome, then the Histogram based technique is more suitable.

\section{ACKNOWLEDGMENT}

We would like to express our gratitude towards our college and our faculty members who guided us throughout our work and kept us motivated.

\section{REFERNCES}

[1] Neha Gupta, V .K. Banga,'Image Segmentation for Text Extraction", April 28-29, 2012.

[2] C.P. Sumathi, T. Santhanam and G.Gayathri Devi",A SURVEY ON VARIOUS APPROACHES OF TEXT EXTRACTION IN IMAGES", August 2012.

[3] E.Chandra"Text Extraction from PDF document" Amrita International Conference of Women in Computing (AICWIC'13) Proceedings published by International Journal of Computer Applications ${ }^{\circledR}$ (IJCA)
[4] Pinky Gather, Avininder Singh,” Empirical Performance Evaluation Methodology and its Application to Page Segmentation Algorithms: A Review", April 2015.

[5] Ankush Gautam," Segmentation of Text From Image Document", 2013.

[6] D.SASIREKHA 1 , Dr.E.CHANDRA," Text Extraction from Pdf Image Using Enhanced Connected Component Labeling”, 1833

[7] Laurence Likforman-Sulem, Abderrazak Zahour, Bruno Taconet," Text Line Segmentation of Historical Documents: a Survey", 2006.

[8] Avinash N Bhute and B.B. Meshram," Text Based Approach For Indexing And Retrieval Of Image And Video: A Review", March 2014

[9] Kewal Krishan, Sukhjit Singh," Color Image Segmentation Using Improved Region Growing and K-Means Method”, May. 2014

[10] Mrunmayee Patil, Ramesh Kagalkar," A Review on Conversion of Image to Text As Well As Speech Using Edge Detection and Image Segmentation", 2012. 